\title{
The impact of orbital prefrontal cortex damage on emotional activation to unanticipated and anticipated acoustic startle stimuli
}

\author{
NICOLE A. ROBERTS \\ University of Wisconsin, Milwaukee, Wisconsin \\ JENNIFER S. BEER \\ University of California, Davis, California \\ KELLY H. WERNER \\ University of California, Berkeley, California \\ DONATELLA SCABINI \\ University of California, Berkeley, California \\ and Helen Wills Neuroscience Institute, Berkeley, California \\ SARA M. LEVENS \\ New York University, New York, New York \\ ROBERT T. KNIGHT \\ University of California, Berkeley, California \\ and Helen Wills Neuroscience Institute, Berkeley, California \\ and \\ ROBERT W. LEVENSON \\ University of California, Berkeley, California
}

\begin{abstract}
Damage to the orbital prefrontal cortex has been implicated in selectively diminishing electrodermal autonomic nervous system responses to anticipated punishing stimuli (e.g., losing money; Bechara, Damasio, \& Damasio, 2000), but not to unanticipated punishing stimuli (e.g., loud noises; Damasio, Tranel, \& Damasio, 1990). We extended this research by examining the effects of orbitofrontal damage on emotional responses to unanticipated and anticipated acoustic startles and collecting a more extensive set of physiological measures, emotional facial behavior, and self-reported emotional experience. Consistent with previous research, patients showed intact physiology to an unanticipated startle but failed to show appropriate anticipatory cardiovascular responses (patients' heart rates decreased, controls' increased). In addition, patients displayed more surprise facial behavior and reported marginally more fear than did controls in response to the unanticipated startle. Thus, orbitofrontal damage may compromise the ability to anticipate physiologically the onset of aversive stimuli, despite intact or enhanced emotional responses when such stimuli occur unexpectedly.
\end{abstract}

The orbital region of the prefrontal cortex (the orbitofrontal cortex) has been implicated in the representation of emotional information and the regulation of emotional processes. The medial region of the orbitofrontal cortex in particular is thought to "influence the affective 'tone' of behavior" (Keay, Clement, \& Bandler, 2000, p. 327). Hypoactivation of the orbitofrontal cortex is associated with such emotional states and behaviors as apathy, in-

This research was supported by NINDS Grants NS 21135 and PO NS40813 to R.T.K. and by NIA Grants AG19724 and AG17766 to R.W.L. Correspondence concerning this article should be addressed to N. A. Roberts, Department of Psychology, University of Wisconsin, P. O. Box 413, Milwaukee, WI 53201 (e-mail: nroberts@uwm.edu). difference, poor planning, and irritability (Horne, 1993). Damage to the orbitofrontal cortex results in socioemotional deficits, including emotional outbursts, impulsivity, risk taking, difficulty with goal-directed behavior, and a failure to abide by social rules and norms (Bechara, Damasio, \& Damasio, 2000; Hartikainen, Ogawa, \& Knight, 2000; Kolb \& Whishaw, 1990; Tucker, Luu, \& Pribram, 1995). In particular, an increasing body of evidence suggests that the orbitofrontal cortex helps link internal emotional cues, such as physiological arousal, with environmental cues, such as reinforcement or punishment (Bechara, Damasio, \& Damasio, 2000; Rolls, $2000)$. For example, patients with damage to the ventromedial region of the orbitofrontal cortex fail to recruit 
anticipatory physiological responses that may guide decision making (Damasio, 1994).

In the present study, we examined the effects of ventromedial orbitofrontal cortex damage on emotional activation in response to powerful emotion-eliciting stimuli (acoustic startles). This study builds on previous research by examining multiple components of emotion, including autonomic (electrodermal and cardiovascular) and somatic nervous system physiology, emotional expressive behavior, and self-reported subjective emotional experience, and by studying both unanticipated and anticipated variants of the same stimulus. Examining multiple measures of emotion is necessary because different components of emotion, and different measures within each component, can reveal different patterns of findings (e.g., Boyce et al., 1993; Gross \& Levenson, 1993; Lacey \& VanLehn, 1952). In particular, neurological disturbance, as evidenced by neurological and clinical populations, may be related to dissociations among emotion components (Kring \& Neale, 1996; Roberts et al., 2001).

\section{The Role of the Orbitofrontal Cortex in Emotion}

Several theories of orbitofrontal functioning have been advanced to explain the frequent observation of pronounced socio-emotional deficits (e.g., disinhibited social behavior or detrimental decision-making strategies) among patients with damage to this region.

Filtering irrelevant emotional information. Shimamura (2000) has proposed that the orbitofrontal cortex helps regulate emotional behavior by gating, or filtering out, irrelevant sensory information that would otherwise be translated into emotional information. Through this type of gating mechanism, the orbitofrontal cortex filters the influence of emotional responses on cognition. In patients with orbitofrontal damage, however, the ability to appropriately gate irrelevant information is inhibited. Thus, according to Shimamura's theory, orbitofrontal patients have difficulty identifying and attending to relevant emotional information and, therefore, show heightened or inappropriate emotional reactions.

Forming and reversing stimulus-response associations. Rolls (2000) has posited that the orbitofrontal cortex plays a role in emotion by helping individuals learn and unlearn stimulus-response associations. Research with both animals and humans suggests that the orbitofrontal cortex assigns meaning to reinforcing cues in the environment (Rolls, 2000; Schoenbaum, Chiba, \& Gallagher, 2000; Schoenbaum \& Setlow, 2001). For example, the orbitofrontal cortex is activated when associations between visual stimuli and taste rewards (or aversive tastes) are learned and then unlearned (i.e., reversed; Rolls, 2000). Thus, according to Rolls, in situations in which reinforcement contingencies rapidly change, such as in social environments, patients with orbitofrontal damage may fail to adjust their behavior appropriately to the demands of the situation.

Generating anticipatory emotional signals. Damasio (1994) has proposed that the orbitofrontal cortex is involved in the generation of physiological reactions that appropriately direct behavior. In a series of studies, Damasio and colleagues demonstrated that neurologically intact subjects show greater electrodermal activity (i.e., skin conductance responses) when they are about to make a risky or potentially disadvantageous decision (e.g., selecting a card from a disadvantageous deck; see Bechara, Damasio, \& Damasio, 2000, for a review). Damasio suggests that these anticipatory physiological responses provide cues, or "hunches," that can be used to guide behavior. Patients with damage to the ventromedial region of the orbitofrontal cortex, however, fail to show such anticipatory physiological responses. As a result, patients are more apt to engage in risky behavior and to make poor decisions (Bechara, Damasio, \& Damasio, 2000; Rahman, Sahakian, Cardinal, Rogers, \& Robbins, 2001).

\section{Orbitofrontal Cortex and Emotional Activation}

Despite theoretical and empirical evidence suggesting the role of the orbitofrontal cortex in emotion-related processes (e.g., filtering irrelevant information, learning and unlearning stimulus-response associations, or anticipating stimuli with uncertain outcomes), less is known about the impact of orbitofrontal cortex damage on emotional activation (i.e., the patterned physiological, expressive, and subjective responses activated to help the organism respond efficiently to changes in the internal and external environment that have significance for goals and well-being; Levenson, 2001). There is some evidence to suggest that damage to the orbitofrontal cortex does not affect the physiological aspect of emotional activation to simple stimuli, such as an unanticipated acoustic startle (Damasio, 1994), that do not require extensive cognitive processing. Because previous studies have not systematically investigated unanticipated and anticipated versions of such simple stimuli, we studied the impact of orbitofrontal damage on emotional activation in response to acoustic startle stimuli that were either unanticipated or anticipated. To ensure a more comprehensive assessment of emotion, we sampled from multiple domains, including cardiovascular, electrodermal, and somatic physiology, expressive facial behavior, and subjective emotional experience.

\section{The Use of Acoustic Startle}

Responses to acoustic startle stimuli are thought to exist on the boundary between reflex (e.g., defensive reactions) and emotion (Ekman, Friesen, \& Simons, 1985). For this reason, startle stimuli are useful for investigating the role of the orbitofrontal cortex in emotion, providing a foundation for subsequent investigations of more complex emotional, cognitive, and social processes.

We presented orbitofrontal patients and controls with brief, loud bursts of white noise of sufficient intensity $(115 \mathrm{~dB})$ to be unambiguously noxious. These startle stimuli elicit stereotyped combinations of physiological, behavioral, and subjective responses, including primary defensive behaviors (e.g., ducking one's head) and secondary emotional responses (e.g., fear and surprise; Ek- 
man et al., 1985; Landis \& Hunt, 1939). As was noted earlier, emotional reactions to these kinds of simple stimuli require much less elaborate cognitive processing, judgment, and decision making than do other emotioneliciting stimuli (e.g., card games or social scenarios).

In its most basic form, the startle stimulus can be presented without warning, which gives subjects minimal opportunity for appraisal or preparation. It can also be presented with warning, which gives subjects time to appraise, anticipate, and react to the impending stimulus. When the startle is presented with warning (e.g., following a visible countdown), it is sufficiently aversive to produce marked anticipatory emotional responses in neurologically intact individuals. Exposing subjects to both types of startles (i.e., unanticipated and anticipated) provides an opportunity to focus on anticipatory reactions in a situation with minimal cognitive demands and in which an aversive outcome is certain.

\section{Overview of the Present Study}

The aim of the present study was to examine the impact of orbitofrontal cortex damage on emotional activation in response to startle stimuli that were either unanticipated or anticipated. Five male patients with bilateral lesions to the ventromedial region of the orbital prefrontal cortex and 5 healthy male control subjects were presented with two acoustic startles: The first one occurred unexpectedly (unanticipated startle), and the second one followed a 20 -sec countdown (anticipated startle). The subjects' physiological (cardiovascular, electrodermal, and somatic), behavioral (facial displays of fear and surprise), and subjective emotional (self-reports of fear and surprise) reactions were examined during and immediately following both startles, as well as during the anticipatory period before the anticipated startle.

\section{Hypotheses}

Hypothesis 1: Reactions to unanticipated startle. We hypothesized that patients and controls would not differ significantly in their (a) physiological reactions (cardiovascular, electrodermal, and somatic), (b) displays of emotional facial behavior (fear and surprise), or (c) selfreported subjective emotional experience (fear and surprise) in response to an unanticipated acoustic startle.

This hypothesis was based on previous research indicating that patients with orbitofrontal damage do not differ significantly from control subjects in their electrodermal reactions to an unanticipated startle (Damasio, 1994).

Hypothesis 2: Reactions to anticipated startle. We hypothesized that patients would (a) show diminished physiological reactions (cardiovascular, electrodermal, and somatic), (b) show fewer displays of emotional facial behavior (fear and surprise), and (c) report less subjective emotional experience (fear and surprise) in response to an anticipated acoustic startle. For the physiological and behavioral measures, we expected that the patients' diminished responding also would be evident during the 20 -sec countdown period (self-report data were not collected during this period, so as not to interrupt the countdown).
This hypothesis was based, in part, on the finding that orbitofrontal patients fail to show anticipatory electrodermal responses prior to the onset of a punishing stimulus (Damasio, 1994) and, in part, on the theory that the orbitofrontal cortex helps individuals learn stimulusresponse associations (Rolls, 2000). Given that all the subjects would experience the unanticipated startle first, we expected the control subjects to retain their negative emotional experience of the first startle and, thus, demonstrate anticipatory physiological increases and facial displays in preparation for the second startle. (Neurologically intact individuals typically show these kinds of anticipatory increases when an aversive stimulus is pending.) In contrast, we expected that the patients would not retain the negative emotional impact of the first startle and, thus, would not show the same preparatory physiological and behavioral increases as controls.

Given our expectation that the patients would not perceive as aversive the fact that the startle noise was going to occur at the end of the countdown, we expected that, in contrast with the controls, the patients would not be in a negative emotional state when the startle noise occurred. Previous research suggests that negative emotional states are associated with heightened startle responses (e.g., Bradley \& Lang, 2000); thus, if the patients were to be in a less aroused state, as compared with controls, before the startle, it follows that they would show smaller responses when the startle occurred.

\section{METHOD}

\section{Subjects}

Five male patients (mean age $=52.4$ years) with bilateral lesions centered in the ventromedial region of the orbital prefrontal cortex and 5 healthy male control subjects (mean age $=60.8$ years) participated in the present study. (A 6th patient participated in the procedure but was excluded from data analyses because his lesion was unilateral.) The patients were recruited from the Northern California Veterans Administration Health Care System, Martinez, California, where they underwent neuropsychological and neuroanatomical evaluations. The patients had incurred focal injuries to the orbitofrontal cortex via head trauma (e.g., a motorcycle accident or falling from a roof). Participation was voluntary, and the patients received $\$ 10$ per hour as compensation. The control subjects were recruited through newspaper advertisements and word of mouth. The controls were not taking medication and did not have neurological or psychiatric conditions. Eleven control subjects participated in the procedure; because some of these subjects were recruited as comparison subjects for a study involving an older cohort (i.e., patients with dementia), data from 5 age-matched controls were analyzed for comparison with the present sample of patients.

Anatomy. In Figure 1, individual axial slices are shown for the 5 patients with lesions encompassing the bilateral orbital prefrontal cortex. Lesion extent was obtained from MRI scans and by utilizing a template system (Rorden \& Brett, 2000). Lesions for the group overlapped in the ventromedial portion of the orbital prefrontal cortex, with extension into the inferior lateral prefrontal cortex in some patients. The patients were comparable in lesion localization to those reported by the research group at the University of Iowa (e.g., Bechara, Damasio, \& Damasio, 2000; Damasio et al., 1990).

\section{Acoustic Startle Stimuli}

Each acoustic startle was a 115-dB, 100-msec burst of white noise administered through two loudspeakers located behind the subject's 


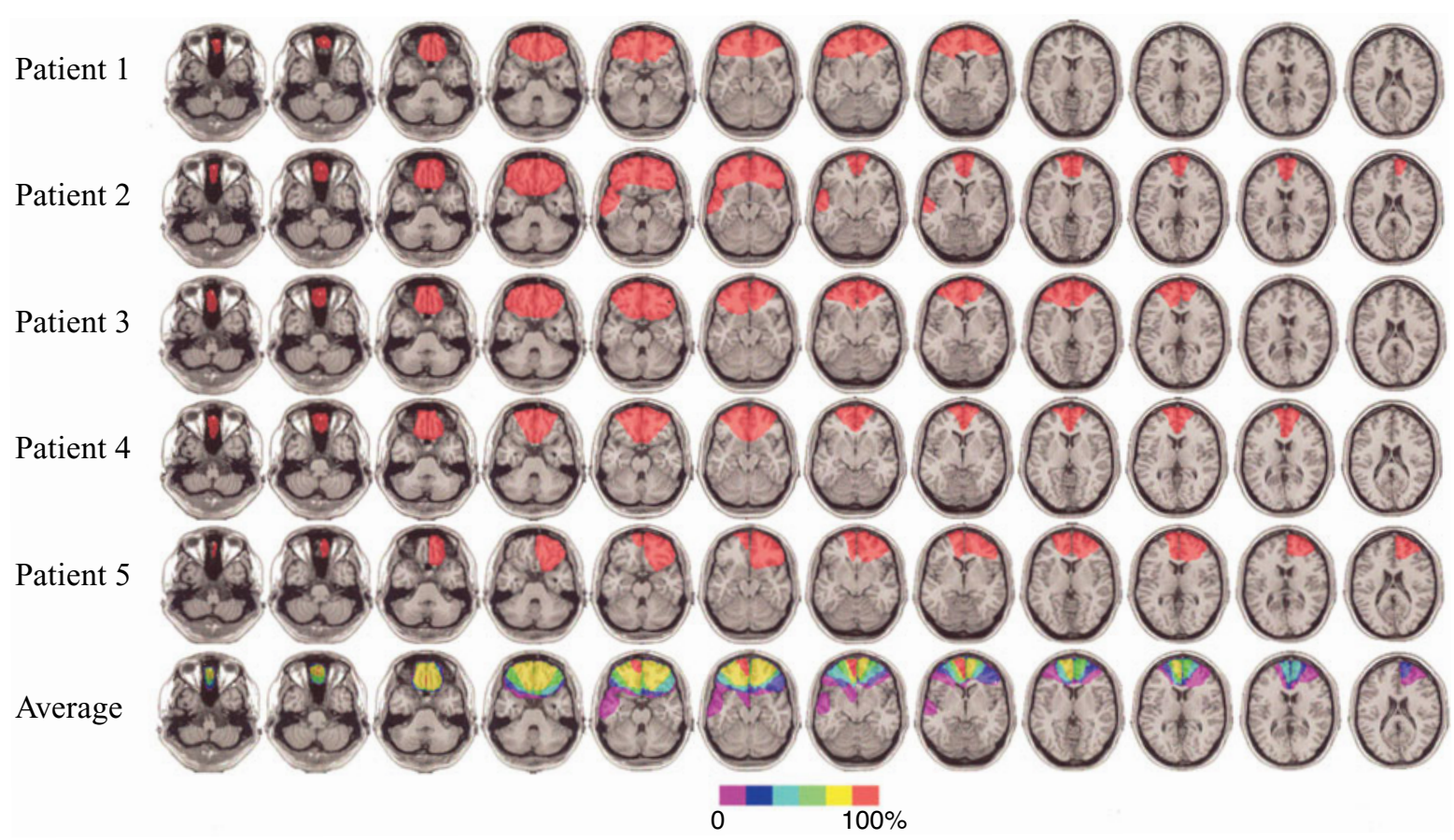

Figure 1. Extent of lesions in orbitofrontal cortex patients $(n=5)$ as reconstructed from MRI or CT scans. Each row shows the extent of damage in an individual patient as transcribed onto axial templates, using 5-mm cuts (lesion marked in red). The bottom row represents average extent of overlap across the 5 patients (percentage of overlap is indicated by color code).

head. The resulting noise was a highly salient and noxious stimulus that simulated a gunshot. It should be noted that this kind of acoustic startle is much louder and longer than the kind of background startle probe stimulus used in contemporary studies of positive and negative affect (e.g., Bradley \& Lang, 2000; Vrana, Spence, \& Lang, 1988).

\section{Apparatus}

Physiological. Three physiological measures were examined in the present study. The first was the cardiac interbeat interval. The electrocardiogram was recorded using Beckman miniature electrodes filled with Redux paste and placed on opposite sides of the chest. The length of the interval between successive R-waves was measured in milliseconds. The second physiological measure was the skin conductance level. To measure the level of sweat gland activity on the surface of the hand, a small constant voltage was passed between Beckman regular electrodes attached to the palmar surface of the lower phalanges of the first and second fingers on the nondominant hand. The electrolyte used was sodium chloride in Unibase. The third physiological measure was general somatic activity. To measure somatic activity, or bodily movement, an electromechanical transducer was attached to a platform under the subject's chair. The transducer generated an electrical signal proportional to the amount of movement in any direction. Somatic activity was measured in arbitrarily designated units. In addition to the transducers used to record these measures, a grounding clip filled with Redux paste was placed on the subject's left ear.

These three physiological measures enabled continuous unobtrusive monitoring of cardiovascular, electrodermal, and somatic activity, all important aspects of emotional activation. These measures allowed us to expand on prior work with patients with orbitofrontal damage, which primarily had examined electrodermal responses (e.g., Damasio, 1994).

Physiological data were collected continuously using a 12-channel Grass Model 7 polygraph and were processed by a microcomputer
}

system using software written by one of the authors (R.W.L.). Secondby-second averages were derived for each measure.

Audiovisual. The subjects received instructions on a $48-\mathrm{cm}$ color television monitor placed on a table $1.25 \mathrm{~m}$ from the subject. A remotely controlled high-resolution video camera partially concealed behind darkened glass and embedded in a bookshelf was used to obtain a frontal view of the subject's face and upper torso in an unobtrusive manner.

Self-reported emotion. After each startle, the subjects completed an inventory (adapted from Ekman, Friesen, \& Ancoli, 1980) that measured self-reported subjective emotional experience. The subjects used 9-point Likert-type scales (anchored by $0=$ not at all, $4=$ moderately, and $8=$ very much) to rate how strongly they felt several specific emotions (including fear and surprise) at the moment when the loud noise occurred.

\section{Procedure}

The subjects completed one 4-h laboratory session. On arrival, the subjects were seated in a comfortable chair in a well-lit $3 \times 6 \mathrm{~m}$ room. Physiological sensors were attached, and the subjects were presented with 15 experimental trials (viewing 10 film clips, posing a silly face, performing an isometric exercise, performing a breathing exercise, and being startled by two loud noises). The focus of the present investigation was on the last two conditions in this session, which each consisted of a single trial (i.e., one startle presentation in each respective trial). ${ }^{1}$

Before each trial, the experimenter verbally presented instructions for the upcoming task, and then these instructions were repeated in written form and read aloud on the video monitor. Baseline data were collected for $1 \mathrm{~min}$ before each trial and for $2 \mathrm{~min}$ after each trial. During these baseline periods, the subjects were asked to relax and stare at a letter " $\mathrm{X}$ " that appeared on the video monitor. ${ }^{2}$ The self-report emotion inventory was administered following each posttrial baseline. At the end of the experiment, the ex- 
perimenter detached the physiological sensors, paid and debriefed the subject, and answered any questions.

Acoustic startle trials. On the unanticipated startle trial, the acoustic startle occurred without warning at the end of the 1-min baseline period. On the anticipated startle trial (which always followed the unanticipated startle trial), the subjects received the following instructions: "In this part of the experiment, you will hear a loud noise. You will know exactly when the loud noise will occur. You will see a countdown from 10 to 0 on the video screen. When you see " 0 " the loud noise will happen. Before beginning the countdown, I want you to relax." After the 1-min baseline period, the following instructions were repeated on the video monitor: "Now you will see a countdown from 10 to 0 . When the timer reaches 0 the loud noise will occur." The countdown lasted for $20 \mathrm{sec}$, with each number presented on the screen for $2 \mathrm{sec}$.

\section{Data Reduction}

Physiology. For each physiological measure (cardiac interbeat interval, skin conductance level, and somatic activity), second-bysecond physiological data were averaged for (1) the 1-min baseline period before each startle, (2) a 15-sec period starting with the startle onset, and (3) for the anticipated startle, the 20-sec countdown prior to the startle. The use of a 15 -sec time window for measuring the responses to the startles was selected to account for differences in the time course of the three physiological measures (e.g., skin conductance responds more slowly than heart rate and somatic activity). For each physiological measure and for each startle condition, change scores were computed by subtracting mean prestartle baseline physiology from mean physiology during the 15-sec startle time window. For the anticipated startle condition, similar scores also were computed for the countdown period by subtracting mean prestartle baseline physiology from mean physiology during the 20 -sec countdown.

Expressive behavior. A team of trained research assistants coded the subjects' expressive behavior from videotapes of each subject's face and upper torso, using a modified version of Gross and Levenson's (1993) Emotional Expressive Behavior coding system (intercoder reliability [alpha] for the full set of codes was .80). Coders were blind as to whether the subject was a patient or a control.

In designing the coding system for the primary behavioral responses to the startle, we relied on previous research (Ekman et al., 1985; Landis \& Hunt, 1939) that has indicated that the initial startle response typically includes seven behaviors: hard eye closure, lip stretch, neck stretch, head jerk, shoulder raise, forward lunge, and torso raise. Coders determined whether each of these seven behaviors was present (coded as 1) or absent (coded as 0 ). A startle behavior composite score was computed by averaging these codes.

Startle stimuli also often generate secondary emotional expressions - specifically, fear and surprise. Coders rated each of these emotions on a second-by-second basis during a period beginning $5 \mathrm{sec}$ before and ending $5 \mathrm{sec}$ after each startle, using a 4-point intensity scale $(0=$ none, $1=$ slight, $2=$ moderate, and $3=$ strong $)$.

For each of the behavioral measures (startle behavior composite score, facial displays of fear, and facial displays of surprise), secondby-second codes were averaged for (1) a 6-sec period starting with the startle onset, and (2) for the anticipated startle, the last $5 \mathrm{sec}$ of the countdown prior to the startle. The use of a 6 -sec time window for measuring the responses to the startles was selected to capture the subjects' maximal display of expressive behavior.

Self-reported emotion. We examined the subjects' self-report ratings of fear and surprise in response to each of the startles.

\section{RESULTS}

\section{Data Analytic Strategy}

To test our hypotheses, we conducted a $2 \times 2$ (group [controls or patients] $\times$ startle type [unanticipated or an- ticipated]) analysis of variance (ANOVA) for each physiological, behavioral, and self-report measure. Group was treated as a between-subjects factor, and startle type was treated as a within-subjects repeated measure. When there were significant interactions of startle type and group, follow-up univariate comparisons were conducted for each startle. One-way analyses of variance were conducted to compare group differences in physiology and behavior during the anticipatory countdown period prior to the anticipated startle (as was noted earlier, there were no self-report data collected during the countdown period). Bonferroni corrections were used for all comparisons to minimize Type I error.

\section{Manipulation Check: Effects of Startle Condition Across Participants}

Before testing our hypotheses, we examined the main effects of startle condition to determine whether the two startle conditions were successful in producing different patterns of responding. We expected the unanticipated startle to produce larger responses than the anticipated startle, because providing the opportunity to anticipate - and thus psychologically prepare for - the startle stimulus has been found to lessen its effects on emotional responding (Ekman et al., 1985; Landis \& Hunt, 1939). Although participants occasionally have been found to habituate rapidly to an anticipated startle when it is presented after an unanticipated startle (Ekman et al., 1985), previous work has shown that anticipated startles typically generate smaller emotional responses than do unanticipated startles, even when startle conditions are counterbalanced (Landis \& Hunt, 1939; Soto, Levenson, \& Ebling, in press).

Consistent with previous research indicating that an unanticipated startle produces larger emotional reactions than does an anticipated startle, we found that the participants rated the unanticipated startle as more surprising $[F(1,8)=69.55, p<.01]$ and more fear inducing $[F(1,8)=22.93, p<.01]$ than the anticipated startle. As compared with the anticipated startle, the unanticipated startle elicited more startle behavior $[F(1,8)=7.64, p<$ $.05]$, more surprise behavior $[F(1,8)=36.00, p<.01]$, and greater increases in somatic activity $[F(1,8)=10.16$, $p<.05]$. There were no differences between the startles in terms of fear behavior elicited $[F(1,8)=1.00$, n.s.], cardiovascular activation [cardiac interbeat interval; $F(1,8)=0.56$, n.s.], or electrodermal activation [skin conductance; $F(1,7)=0.57$, n.s.].

\section{Group Differences in Reactions to Unanticipated and Anticipated Acoustic Startle}

Physiological activation. Mean levels of physiology before, during, and after each startle and change scores reflecting physiological activation in response to each startle (i.e., startle physiology minus baseline physiology) are displayed by group in Tables 1 and 2 for the two respective startle conditions.

To test the hypotheses that the patients and the controls would show similar amounts of physiological activation (i.e., similar increases in physiology) to the unantici- 
Table 1

Mean Physiological Responses to Unanticipated Startle (With Standard Deviations)

\begin{tabular}{|c|c|c|c|c|c|c|c|c|}
\hline \multirow[b]{2}{*}{ Measure } & \multicolumn{2}{|c|}{$\begin{array}{c}\text { Prestartle } \\
\text { 1-Min Baseline }\end{array}$} & \multicolumn{2}{|c|}{ Startle: Mean } & \multicolumn{2}{|c|}{$\begin{array}{c}\text { Startle: } \\
\text { Change From Baseline } \\
\end{array}$} & \multicolumn{2}{|c|}{$\begin{array}{c}\text { Poststartle } \\
\text { 2-Min Baseline }\end{array}$} \\
\hline & $M$ & $S D$ & $M$ & $S D$ & $M$ & $S D$ & $M$ & $S D$ \\
\hline \multicolumn{9}{|c|}{ Controls } \\
\hline IBI & 818.7 & 130.5 & 809.7 & 115.4 & -9.0 & 75.7 & 848.9 & 139.2 \\
\hline SCL & 2.2 & 1.3 & 3.0 & 1.6 & 0.9 & 0.4 & 2.6 & 1.2 \\
\hline ACT & 0.9 & 0.1 & 1.6 & 1.1 & 0.7 & 1.1 & 0.9 & 0.2 \\
\hline \multicolumn{9}{|c|}{ Patients } \\
\hline IBI & 858.3 & 56.5 & 835.1 & 54.1 & -23.3 & 65.8 & 886.2 & 35.8 \\
\hline SCL & 3.3 & 2.0 & 4.4 & 2.6 & 1.0 & 0.7 & 4.0 & 2.0 \\
\hline $\mathrm{ACT}$ & 1.5 & 1.2 & 2.3 & 1.3 & 0.7 & 1.4 & 1.7 & 1.5 \\
\hline
\end{tabular}

Note-Startle values reflect a 15 -sec time window beginning with the startle noise. Change from baseline values reflect the mean of the time window of interest (i.e., the 15 -sec startle window) minus the mean of the baseline period. For cardiac interbeat interval, smaller values reflect greater physiological activation; thus, negative change scores represent increases in cardiovascular activation, and positive change scores represent decreases in cardiovascular activation. IBI, cardiac interbeat interval; SCL, skin conductance level; ACT, somatic activity.

pated startle (Hypothesis 1) but that the patients would show less physiological activation than the controls to the anticipated startle (Hypothesis 2), we examined the interaction of group (patient or control) and startle condition (unanticipated or anticipated) for each physiological measure. There was a significant interaction of group and startle condition for cardiac interbeat interval $[F(1,8)=$ $6.88, p<.05]$. Follow-up comparisons revealed that, as was hypothesized, the patients and the controls did not differ significantly in their cardiovascular responses to the unanticipated startle $[F(1,8)=0.10$, n.s.], but that the patients showed diminished cardiovascular activation, as compared with the controls, in response to the anticipated startle $[F(1,8)=5.56, p<.05]$. Specifically, the control subjects showed decreases in cardiac interbeat interval (indicating greater cardiovascular activation) in response to the anticipated startle, whereas the patients showed increases in cardiac interbeat interval (indicating less cardiovascular activation).
The interactions of group and startle condition were not significant for skin conductance level $[F(1,7)=$ 0.10, n.s.] or somatic activity $[F(1,8)=1.30$, n.s.].

To assess differences between the patients and the controls during the countdown period prior to the anticipated startle, one-way ANOVAs were conducted for each physiological measure. These analyses revealed a significant difference between the patients and the controls for cardiac interbeat interval $[F(1,8)=6.41, p<.05]$; the control subjects showed decreases in cardiac interbeat interval (indicating greater cardiovascular activation), whereas the patients showed increases in cardiac interbeat interval (indicating less cardiovascular activation). There were no group differences in skin conductance level $[F(1,7)=$ 0.56, n.s.] or somatic activity $[F(1,8)=1.00$, n.s. $]$ during the countdown.

It should be noted that there were no group differences in physiology during the 1-min baseline period prior to either of the startles or during the 2-min recovery period

Table 2

Mean Physiological Responses to Anticipated Startle (With Standard Deviations)

\begin{tabular}{|c|c|c|c|c|c|c|c|c|c|c|c|c|}
\hline \multirow[b]{2}{*}{ Measure } & \multicolumn{2}{|c|}{$\begin{array}{l}\text { Prestartle } \\
\text { 1-Min } \\
\text { Baseline }\end{array}$} & \multicolumn{2}{|c|}{$\begin{array}{l}\text { Countdown: } \\
\text { Mean }\end{array}$} & \multicolumn{2}{|c|}{$\begin{array}{l}\text { Countdown: } \\
\text { Change From } \\
\text { Baseline }\end{array}$} & \multicolumn{2}{|c|}{$\begin{array}{l}\text { Startle: } \\
\text { Mean }\end{array}$} & \multicolumn{2}{|c|}{$\begin{array}{c}\text { Startle: } \\
\text { Change From } \\
\text { Baseline }\end{array}$} & \multicolumn{2}{|c|}{$\begin{array}{l}\text { Poststartle } \\
\text { 2-Min } \\
\text { Baseline }\end{array}$} \\
\hline & $M$ & $S D$ & $M$ & $S D$ & $M$ & $S D$ & $M$ & $S D$ & $M$ & $S D$ & $M$ & $S D$ \\
\hline \multicolumn{13}{|c|}{ Controls } \\
\hline IBI & 839.4 & 142.1 & 812.0 & 139.6 & $-27.4^{*}$ & 12.1 & 803.9 & 118.6 & $-35.5^{*}$ & 36.8 & 836.3 & 132.7 \\
\hline SCL & 2.1 & 1.2 & 2.4 & 1.1 & 0.3 & 0.5 & 2.9 & 1.2 & 0.8 & 0.6 & 2.5 & 1.1 \\
\hline ACT & 0.8 & 0.2 & 0.8 & 0.2 & 0.0 & 0.0 & 1.0 & 0.4 & 0.2 & 0.3 & 0.8 & 0.2 \\
\hline \multicolumn{13}{|c|}{ Patients } \\
\hline IBI & 864.5 & 67.0 & 877.0 & 64.4 & $11.5^{*}$ & 32.2 & 888.9 & 60.5 & $24.4 *$ & 43.4 & 865.2 & 55.2 \\
\hline SCL & 3.2 & 1.6 & 3.3 & 1.4 & 0.1 & 0.4 & 4.1 & 1.9 & 0.8 & 0.6 & 3.5 & 1.6 \\
\hline ACT & 1.8 & 1.6 & 1.5 & 1.3 & -0.3 & 0.6 & 1.5 & 1.7 & -0.3 & 0.9 & 1.7 & 1.4 \\
\hline
\end{tabular}

Note-Startle values reflect a 15 -sec time window beginning with the startle noise. The countdown is the 20-sec anticipatory period before the startle noise. Change from baseline values reflect the mean of the time window of interest (i.e., the countdown or the startle period) minus the mean of the baseline period. For cardiac interbeat interval, smaller values reflect greater physiological activation; thus, negative change scores represent increases in cardiovascular activation, and positive change scores represent decreases in cardiovascular activation. Asterisks indicate a significant group difference between the two means in that column of the table. IBI, cardiac interbeat interval; SCL, skin conductance level; ACT, somatic activity. ${ }^{*} p<.05$. 
Table 3

Mean Amount of Expressive Behavior Displayed in Response to Acoustic Startles (With Standard Deviations)

\begin{tabular}{|c|c|c|c|c|}
\hline \multirow[b]{2}{*}{ Measure } & \multicolumn{2}{|c|}{ Control Subjects } & \multicolumn{2}{|c|}{ Orbitofrontal Patients } \\
\hline & $M$ & $S D$ & $M$ & $S D$ \\
\hline \multicolumn{5}{|c|}{ Unanticipated Startle (6-sec Startle Time Window) } \\
\hline Startle behavior & 2.0 & 1.9 & 2.6 & 1.7 \\
\hline Surprise behavior & $0.5^{*}$ & 0.5 & $1.2 *$ & 0.3 \\
\hline Fear behavior & 0.0 & 0.0 & 0.3 & 0.4 \\
\hline \multicolumn{5}{|c|}{ Anticipated Startle (6-sec Startle Time Window) } \\
\hline Startle behavior & 1.0 & 1.2 & 0.4 & 0.9 \\
\hline Surprise behavior & 0.3 & 0.4 & 0.2 & 0.3 \\
\hline Fear behavior & 0.0 & 0.0 & 0.2 & 0.3 \\
\hline \multicolumn{5}{|c|}{ Anticipated Startle Countdown (5-sec Prestartle) } \\
\hline Surprise behavior & 0.0 & 0.0 & 0.0 & 0.0 \\
\hline Fear behavior & 0.0 & 0.0 & 0.0 & 0.0 \\
\hline
\end{tabular}

Note-Startle behaviors were coded as present (1) or absent (0). Fear and surprise were coded on an intensity scale ranging from 0 (none at all) to 3 (strong displays of behavior). Asterisks indicate a significant difference between the two means in that row of the table. $* p<.05$.

following the unanticipated startle. Thus, group differences in cardiovascular activation in response to the countdown and in response to the anticipated startle are not attributable simply to group differences in recovery from the unanticipated startle.

Expressive behavior. The amount of startle behavior, fear, and surprise displayed by the patients and the controls for each startle condition are presented in Table 3. To test the hypothesis that the patients and the controls would show similar amounts of surprise facial behavior and fear facial behavior in response to the unanticipated startle (Hypothesis 1) but that, in response to the anticipated startle, the patients would show fewer facial displays of surprise and fear behavior than the controls (Hypothesis 2), we examined the interaction of group and startle condition for each emotional behavior. There was a significant interaction of group and startle condition for displays of surprise $[F(1,8)=16.00, p<.01]$. Contrary to our predictions, the patients displayed more surprise than did the controls in response to the unanticipated startle $[F(1,8)=7.54, p<.05]$, and the patients and the controls displayed similar amounts of surprise in response

Table 4

Mean Ratings of Self-Reported Emotion in Response to Acoustic Startles (With Standard Deviations)

\begin{tabular}{|c|c|c|c|c|}
\hline \multirow[b]{2}{*}{ Measure } & \multicolumn{2}{|c|}{ Control Subjects } & \multicolumn{2}{|c|}{ Orbitofrontal Patients } \\
\hline & $M$ & $S D$ & $M$ & $S D$ \\
\hline \multicolumn{5}{|c|}{ Unanticipated Startle } \\
\hline Surprise & 7.6 & 0.5 & 7.6 & 0.5 \\
\hline Fear & $3.0 *$ & 2.0 & $6.2 *$ & 2.7 \\
\hline \multicolumn{5}{|c|}{ Anticipated Startle } \\
\hline Surprise & 1.4 & 1.7 & 1.6 & 2.6 \\
\hline Fear & 0.8 & 1.1 & 1.8 & 2.7 \\
\hline
\end{tabular}

Note-Self-report ratings were made on 9-point scales ranging from 0 (not at all) to 8 (very much). Asterisks indicate a significant difference between the two means in that row of the table. ${ }^{*} p<.07$. to the anticipated startle $[F(1,8)=0.18$, n.s. $]$. The interaction of group and startle condition was not significant for displays of fear behavior $[F(1,8)=1.00$, n.s.].

None of the subjects displayed surprise behavior or fear behavior during the countdown prior to the anticipated startle; therefore, further comparisons between the patients and the controls were not conducted.

Self-reported emotional experience. Mean ratings of self-reported surprise and fear for the patients and the controls for each startle condition are presented in Table 4. Although we expected that the patients and the controls would not differ in their self-reports of surprise or fear in response to the unanticipated startle (Hypothesis 1) but that the patients would report less surprise and fear than the controls in response to the anticipated startle (Hypothesis 2), we found that the interactions of group and startle condition for self-reported surprise $[F(1,8)=$ 0.02$, n.s. $]$ and self-reported fear $[F(1,8)=2.55, p<.15]$ were not significant. However, because the $F$ values for self-reported fear indicated a moderate effect for this sample size (partial $\eta^{2}=0.24$ ), we conducted follow-up univariate analyses for each startle. These analyses revealed a trend toward more fear being reported by the patients than by the controls in response to the unanticipated startle $[F(1,8)=4.57, p<.07]$.

\section{DISCUSSION}

The aim of this study was to examine emotional activation among patients with bilateral damage to the ventromedial region of the orbital prefrontal cortex. We compared the emotional reactions of patients and control subjects in response to relatively simple acoustic startle stimuli that were either unanticipated or anticipated. This study extended previous research on the impact of orbitofrontal cortex damage on anticipatory responses by including a more comprehensive assessment of emotional activation (cardiovascular, electrodermal, and somatic physiology, facial expressions, and subjective emotional responding) and by not confounding the presence or absence of anticipation with the type of stimulus used (e.g., comparing unanticipated startle responses with anticipated losses in a card game).

\section{Reactions to Unanticipated Acoustic Startle \\ Intact physiological, subjective, and primary star-} tle reactions. The unanticipated startle was an equally powerful stimulus for both the patients and the control subjects. In response to the unanticipated startle, the patients and the controls did not differ in their self-reported experience of surprise, primary startle behavior (e.g., hard eye closure, head jerk, or torso raise), somatic activity (e.g., how much they moved when the startle occurred), cardiovascular activation, or electrodermal activation. These findings indicate that a sudden, loud, unexpected acoustic startle is an effective stimulus for eliciting emotion even among patients with significant amounts of orbitofrontal damage. 
Previous findings have shown that electrodermal responses to unanticipated startles remain intact despite focal lesions in the orbitofrontal cortex (Damasio et al., 1990). With these new findings, it appears that this preservation of responding extends to cardiovascular, somatic, primary behavioral, and subjective reactions as well.

Larger emotional reactions. Against this backdrop of similarity of response, the findings that the orbitofrontal patients showed more facial displays of surprise and reported feeling more fear in response to the unanticipated startle than did the control subjects are particularly interesting. It is important to note that displaying surprise and experiencing fear in response to an unanticipated startle are appropriate responses. Thus, this is not an instance of patients showing a peculiar idiosyncratic response but, rather, a difference in amount. Our findings of greater self-reported fear and greater surprise behavior for patients are consistent with the findings of Rule, Shimamura, and Knight (2002), which showed that patients with orbitofrontal damage show central nervous system disinhibition. Our findings also lend support to the notion that the orbitofrontal cortex may act as a filtering system for sensory inputs (Shimamura, 2000). The patients' greater reports of fear and displays of surprise behavior, as compared with the controls, may reflect a kind of behavioral disinhibition, where the patients showed more of an appropriate emotional response when confronted with a sudden powerful stimulus that provided minimal opportunity to exert voluntary or effortful control over their emotional reactions.

Summary of reactions to unanticipated startle. Taken together, the patients' reactions to the unanticipated startle revealed that their basic emotional reactions were intact. However, there is some indication of disinhibition and a pattern of dissociation among the components of emotion: the patients showed intact physiological reactions and primary behavioral startle responses but showed greater displays of surprise facial behavior than did controls and possibly experienced more fear.

\section{Reactions to Anticipated Acoustic Startle}

After presenting the participants with an unanticipated acoustic startle, they were informed that the loud noise would occur again but that they would know exactly when the noise would occur (i.e., at the end of a 20 -sec countdown). The startle stimulus is sufficiently intense that, when warned, participants typically go to considerable lengths to prepare themselves and reduce the impact of the second occurrence. Thus, this paradigm provides an opportunity to compare patients' and controls' anticipatory reactions with a stimulus that makes relatively minimal cognitive demand and when the aversive outcome is certain. Although this study design presented a confound, in that the unanticipated startle was always presented first, this consistent presentation order made it possible to examine group differences in anticipation when the aversive nature of the stimulus was clearly established.

Diminished cardiovascular activation. We found significant differences in the cardiovascular responses of the patients and the control subjects during the countdown and in response to the anticipated startle. Specifically, in both periods, the control subjects showed increases in heart rate, but the patients showed decreases in heart rate. The increases in heart rate in anticipation of and in reaction to aversive events shown by controls are commensurate with defensive reactions associated with emotions such as fear (Cook \& Turpin, 1997; Lang, Bradley, \& Cuthbert, 1997). These physiological changes are often characterized as being part of the fight or flight response seen in reaction to aversive stimuli (e.g., the acoustic startle). The orbitofrontal patients showed a quite different cardiovascular response, consisting of decreases in heart rate in anticipation of and in reaction to the anticipated startle. These kinds of heart rate decreases are typically associated with the orienting response (e.g., Cook \& Turpin, 1997). Thus, the cardiovascular responses of the controls were consistent with their mobilizing physiological resources to deal with an impending noxious stimulus, whereas those of the patients were consistent with their attending to the stimulus. This implies that orbitofrontal cortex damage may interfere with the normal mobilization of cardiovascular resources necessary to respond properly to warnings that an aversive event is imminent. Of note, the patients' pattern of decreased cardiovascular activation during the countdown may have influenced their cardiovascular responses to the startle itself. This suggests that the process of anticipation and, specifically, deficits in this process can influence responding when the anticipated stimulus finally occurs.

Although cardiovascular responses normally follow closely the demands created by activity of the large muscles (Obrist, Webb, Sutterer, \& Howard, 1970), we did not find the same pattern of differences between the patients and the controls for somatic activity as we did for heart rate. Both the patients and the controls showed little change or a slight decrease in somatic activity during the countdown prior to the startle. For the patients, this may have, in fact, reflected a lack of mobilization (consistent with their decreasing heart rate), whereas for the controls it may have reflected a momentary bracing against the impending noxious event prior to taking action (consistent with their increasing heart rate).

Why not diminished electrodermal activation? Although our findings in the realm of cardiovascular activity corroborate previous research that patients fail to show anticipatory physiological responses to punishing stimuli (Damasio, 1994), we did not replicate previous findings that this deficit is found in electrodermal activity. In our sample, the patients and the controls did not differ in their electrodermal reactions during the countdown or in response to the anticipated startle. One possible explanation for this difference in findings is the nature of the tasks or contexts in which the anticipatory responses occurred. Bechara, Tranel, and Damasio (2000) found that orbitofrontal patients showed deficits in electrodermal responding in cognitively complex situations involving a great deal of uncertainty (i.e., the potential loss of money in a gambling task). In contrast, the anticipated 
startle task used in the present study ensures a certain, aversive outcome (i.e., that a loud noise will be sounded at the end of a countdown). In contrast with the complexities and uncertainties inherent in a gambling task, our startle task more closely resembles a fear-conditioning paradigm, which may be influenced more by the amygdala than by the orbitofrontal cortex (Bechara, Damasio, Damasio, \& Lee, 1999; Phelps et al., 2001; Quirk, Armony, \& LeDoux, 1997). In addition, whereas the gambling task involves a social, or abstract, consequence (i.e., threat of financial loss), the anticipated startle involves a physical consequence (i.e., threat of bodily harm). Thus, our findings suggest that the orbitofrontal cortex is not necessarily implicated in generating arousal responsesand specifically, electrodermal activation - to physically threatening stimuli but, rather, may be implicated in generating arousal responses to social stimuli. This is consistent with findings suggesting that the orbitofrontal cortex is involved in facilitating socially appropriate behavior (Beer, Heerey, Keltner, Scabini, \& Knight, 2003).

Dissociation among autonomic nervous system measures. The fact that in response to an anticipated startle, orbitofrontal patients and controls did not differ significantly in their electrodermal responses, yet the patients showed smaller cardiovascular responses than did the controls (and specifically, cardiovascular decreases rather than increases) points to a dissociation among autonomic nervous system measures. These kinds of dissociations are not uncommon among neurological, as well as clinical, populations (e.g., Fowles, 1980; Maschke et al., 2002). Fowles (1980) has proposed that cardiovascular activation is associated with behavioral activation (e.g., active avoidance) and electrodermal activation is associated with behavioral inhibition (e.g., passive avoidance). Thus, orbitofrontal patients may have some understanding that the pending startle stimulus is aversive and, hence, do not differ from controls in terms of their anticipatory electrodermal responses. However, they may not be mobilized for action to deal purposefully and effectively with the noxious event, as is demonstrated by their lack of heart rate increases.

Intact expressive behavior and subjective emotional experience. We had expected that, along with diminished anticipatory physiological responses, patients would show fewer displays of emotion and report feeling less emotion than controls would in response to the anticipated startle. However, there were no group differences in facial displays of fear or surprise prior to the anticipated startle (in fact, neither the controls nor the patients showed facial displays of fear or surprise during the anticipation period), nor were there group differences in facial displays or self-reports of fear or surprise during and immediately after the anticipated startle. As with the unanticipated startle, it appears that these behavioral and subjective aspects of emotional activation to the anticipated startle remain intact in orbitofrontal patients.

Summary of reactions to anticipated startle. As was the case for the unanticipated startle, the patients' emotional responses prior to, as well as during, the an- ticipated startle revealed a pattern of dissociation across components of emotion (i.e., physiology vs. behavioral expression and self-reported subjective experience) and even within the physiological component of emotion (i.e., cardiovascular vs. electrodermal and somatic activation). In response to a simple, forewarned noxious stimulus, most aspects of the emotional response remained intact in the orbitofrontal patients. However, the patients showed a pattern of cardiovascular response that suggested they were merely orienting to the noxious event, and not mobilizing the resources to deal with it in an efficient way. Such an interpretation is consistent with Rolls (2000), in that it may reflect a failure on the part of the orbitofrontal patients to learn from the emotional impact of the first unanticipated startle and to make associations that would prepare them for dealing with the second anticipated startle.

\section{Conclusions About the Role of the Orbitofrontal Cortex in Emotion}

Our findings shed additional light on the role that the orbitofrontal cortex plays in shaping emotional responses. It would be an overgeneralization to implicate the orbitofrontal cortex as a region responsible for emotion. Even with bilateral orbitofrontal lesions, the patients were able to show emotional responses to powerful aversive stimuli that had many of the same physiological, behavioral, and subjective features as the responses of the controls. Nevertheless, in the cases of both the unanticipated and the anticipated startles, subtle differences were revealed. In response to the unanticipated startle, the patients showed more facial displays of surprise and reported feeling marginally more fear than did the controls, suggesting some disinhibition of response. In response to the anticipated startle, the patients showed a deceleration of heart rate consistent with orienting to the stimulus, whereas the controls showed an acceleration of heart rate consistent with mobilizing resources to deal with the aversive event. To the extent that autonomic nervous system activation provides cues that guide subsequent responses (Damasio, 1994), with the diminishing of these responses (cardiovascular in our study, electrodermal in Damasio's studies), patients with orbitofrontal lesions may be left without important physiological cues necessary to facilitate rapid and appropriate responses to threatening situations.

Our findings also suggest that orbitofrontal patients have a degree of dissociation among the physiological, behavioral, and subjective aspects of emotion, as compared with controls. In the case of the unanticipated startle, the patients' physiological reactions were similar to those of the controls, but their facial expressions and subjective experiences were exaggerated. In the case of the anticipated startle, the patients had similar facial displays of emotion as controls but had an unconventional pattern of cardiovascular activity (i.e., heart rate decreases rather than increases). With such deviations from the normal "tuning" of the emotional response, it seems reasonable to expect orbitofrontal patients to demonstrate inappropriate socio-emotional behavior that would be noticed by 
friends, family, clinicians, and researchers. Moreover, these deviations in emotional response can be expected to have an adverse impact on such cognitive processes as attention, learning, memory, and decision making, all of which are profoundly influenced by emotional reactions.

\section{REFERENCES}

Bechara, A., Damasio, H., \& Damasio, A. R. (2000). Emotion, decision making, and the orbitofrontal cortex. Cerebral Cortex, 10, 295-307.

Bechara, A., Damasio, H., Damasio, A. R., \& Lee, G. P. (1999). Different contributions of the human amygdala and ventromedial prefrontal cortex to decision-making. Journal of Neuroscience, 19 , 5473-5481.

Bechara, A., Tranel, D., \& Damasio, H. (2000). Characterization of the decision-making deficit of patients with ventromedial prefrontal cortex lesions. Brain, 123, 2189-2202.

Beer, J. S., Heerey, E. A., Keltner, D., Scabini, D., \& Knight, R. T. (2003). The regularity function of self-conscious emotion: Insights from patients with orbitofrontal damage. Journal of Personality \& Social Psychology, 85, 594-604.

Boyce, W. T., Chesterman, E. A., Martin, N., Folkman, S., Cohen, F., $\&$ WARA, D. (1993). Immunologic changes occurring at kindergarten entry predict respiratory illnesses after the Loma Prieta earthquake. Journal of Developmental \& Behavioral Pediatrics, 14, 296-303.

BRADLEY, M. M., \& LANG, P. J. (2000). Affective reactions to acoustic stimuli. Psychophysiology, 37, 204-215.

CoOK, E., \& TurPIN, G. (1997). Differentiating orienting, startle, and defense responses: The role of affect and its implications for psychopathology. In P. J. Lang, R. F. Simons, \& M. T. Balaban (Eds.), Attention and orienting: Sensory and motivational processes (pp. 137164). Mahwah, NJ: Erlbaum.

Damasio, A. R. (1994). Descartes' error: Emotion, reason, and the human brain. New York: Putnam.

Damasio, A. R., Tranel, D., \& Damasio, H. (1990). Individuals with sociopathic behavior caused by frontal damage fail to respond autonomically to social stimuli. Behavioural Brain Research, 41, 81-94.

EkMAN, P., Friesen, W. V., \& ANCOLI, S. (1980). Facial signs of emotional experience. Journal of Personality \& Social Psychology, 39, 1125-1134.

Ekman, P., Friesen, W. V., \& SimONS, R. C. (1985). Is the startle reaction an emotion? Journal of Personality \& Social Psychology, 49, 1416-1426.

FowLES, D. C. (1980). The three arousal model: Implications of Gray's two-factor learning theory for heart rate, electrodermal activity, and psychopathy. Psychophysiology, 17, 87-103.

Gross, J. J., \& LeVEnson, R. W. (1993). Emotional suppression: Physiology, self-report, and expressive behavior. Journal of Personality \& Social Psychology, 64, 970-986.

Hartikainen, K. M., OGaWA, K. H., \& Knight, R. T. (2000). Transient interference of right hemispheric function due to automatic emotional processing. Neuropsychologia, 38, 1576-1580.

Horne, J. A. (1993). Human sleep, sleep loss and behavior: Implications for the prefrontal cortex and psychiatric disorder. British Journal of Psychiatry, 162, 413-419.

KeAY, K. A., Clement, C. I., \& BANDLER, R. (2000). The neuroanatomy of cardiac nociceptive pathways. In G. J. Ter Horst (Ed.), The nervous system and the heart (pp. 303-342). Totowa, NJ: Humana.

Kolb, B., \& Whishaw, I. Q. (1990). The frontal lobes. In B. Kolb \& I.Q. Whishaw (Eds.), Fundamentals of human neuropsychology (3rd ed., pp. 463-501). New York: Freeman.

KrING, A. M., \& Neale, J. M. (1996). Do schizophrenic patients show a disjunctive relationship among expressive, experiential, and psychophysiological components of emotion? Journal of Abnormal Psychology, 105, 249-257.

LACEY, J. I., \& VANLEHN, R. (1952). Differential emphasis in somatic response to stress: An experimental study. Psychosomatic Medicine, 14, 71-81.

LANDIs, C., \& HunT, W. A. (1939). The startle pattern. New York: Farrar \& Rinehart.

Lang, P. J., Bradley, M. M., \& Cuthbert, B. N. (1997). Motivated at- tention: Affect, activation, and action. In P. J. Lang, R. F. Simons, \& M. T. Balaban (Eds.), Attention and orienting: Sensory and motivational processes (pp. 97-135). Mahwah, NJ: Erlbaum.

LEVENSON, R. W. (2001). Emotional functioning in frontotemporal lobar degeneration. Unpublished manuscript, University of California, Berkeley, Department of Psychology.

Maschie, M., Schugens, M., Kindsvater, K., Drepper, J., Kolb, F. P., Diener, H. C., Daum, I., \& Timmann, D. (2002). Fear conditioned changes of heart rate in patients with medial cerebellar lesions. Journal of Neurology, Neurosurgery, \& Psychiatry, 72, 116-118.

OBrist, P. A., WebB, R. A., SutTerer, J. R., \& Howard, J. L. (1970). The cardiac-somatic relationship: Some reformulations. Psychophysiology, 6, 569-587.

Phelps, E. A., O’Connor, K. J., Gatenby, J. C., Gore, J. C., Grillon, C., \& Davis, M. (2001). Activation of the left amygdala to a cognitive representation of fear. Nature Neuroscience, 4, 437-441.

Quirk, G. J., Armony, J. L., \& LeDouX, J. E. (1997). Fear conditioning enhances different temporal components of tone-evoked spike trains in auditory cortex and lateral amygdala. Neuron, 19, 613-624.

Rahman, S., Sahakian, B. J., Cardinal, R. N., Rogers, R. D., \& RobBINS, T. W. (2001). Decision making and neuropsychiatry. Trends in Cognitive Sciences, 5, 271-277.

Roberts, N. A., Werner, K. H., Beer, J. S., LeVenson, R. W., Rosen, H. J., Perry, R. J., \& Miller, B. L. (2001, October). Cerebral lateralization of emotion in frontotemporal lobar dementia. Poster presented at the annual meeting of the Society for Psychophysiological Research, Montreal.

Rolls, E. T. (2000). The orbitofrontal cortex and reward. Cerebral Cortex, 10, 284-294.

Rorden, C., \& Brett, M. (2000). Stereotaxic display of brain lesions. Behavioural Neurology, 12, 191-200.

Rule, R. R., Shimamura, A. P., \& KNight, R. T. (2002). Orbitofrontal cortex and dynamic filtering of emotional stimuli. Cognitive, Affective, \& Behavioral Neuroscience, 2, 264-270.

Schoenbaum, G., Chiba, A. A., \& Gallagher, M. (2000). Changes in functional connectivity in orbitofrontal cortex and basolateral amygdala during learning and reversal training. Journal of Neuroscience, 20, 5179-5189.

ScHOENBAUM, G., \& SeTLOW, B. (2001). Integrating orbitofrontal cortex into prefrontal theory: Common processing themes across species and subdivisions. Learning \& Memory, 8, 134-147.

Shimamura, A. (2000) The role of the prefrontal cortex in dynamic filtering. Psychobiology, 28, 207-218.

Soto, J. A., Levenson, R. W., \& Ebling, R. (in press). Cultures of moderation and expression: Emotional experience, behavior, and physiology in Chinese Americans and Mexican Americans. Emotion.

TuCKer, D. M., LuU, P., \& Pribram, K. H. (1995). Social and emotional self-regulation. In J. Grafman, K. J. Holyoak, \& F. Boller (Eds.), Structure and function of the human prefrontal cortex (Annals of the New York Academy of Sciences, Vol. 769, pp. 213-239). New York: New York Academy of Sciences.

VRANA, S. R., SPEnce, E. L., \& LANG, P. J. (1988). The startle probe response: A new measure of emotion? Journal of Abnormal Psychology, 97, 487-491.

\section{NOTES}

1. Although the subjects may have experienced some fatigue by the end of the experiment, the startle is a sufficiently powerful stimulus to elicit physiological, behavioral, and subjective emotional responses, regardless of possible fatigue effects (Landis \& Hunt, 1939). In addition, we do not have reason to suspect that the patients and the controls in this sample would be differentially fatigued by the experiment.

2. Although physiological responses to an acoustic startle may be captured by a relatively brief time window (i.e., $15 \mathrm{sec}$ ), 2 min of physiological data were collected after each startle to determine whether there were group differences in the time it takes for subjects to physiologically recover (i.e., return to baseline) following the startle noise.

(Manuscript received February 28, 2003; revision accepted for publication May 24, 2004.) 